\title{
Staging of Hepatocellular Carcinoma Using Deep Feature in Contrast- Enhanced MR Images
}

\author{
Qiyao Wang 1, a , Dashun Que 1, 2, b \\ ${ }^{1}$ School of Information Engineering, Wuhan University of Technology, Wuhan, 430070, China. \\ ${ }^{2}$ Key Laboratory of Fiber Optic Sensing Technology and Information Processing, Ministry of Edu- \\ cation, Wuhan University of Technology, Wuhan, 430070, China. \\ a332102691@qq.com, bdsque@sina.com
}

Keywords: Hepatocelluler Carcinoma, Deep Feature, Convolutional Neural Network, Ensemble.

\begin{abstract}
Clinical stage of hepatocellular carcinoma (HCC) is of great significance for prognosis. Texture features of HCC in Contrast-enhanced MR images have been effective for predictions of staging. However, texture features are low-level features, which are usually insufficient to capture the complicated characteristics of HCCs. Recently, some studies have been dedicated to learning features in a data driven way for predictions. In this study, we use deep learning that can extract highlevel features in order to more accurately staging HCCs. Experimental results demonstrate that deep feature outperforms traditional texture features for HCC staging, and ensembles of deep features derived from multiview observations of HCCs yield best results.
\end{abstract}

\section{Introduction}

Hepatocellular carcinoma (HCC) is the third largest cancer in the world. The histological grade of HCC differentiation has significance in establishing good treatment strategy. Recently, Texture features of liver tumor CT images have also been shown to be associated with tumor staging. Deep features obtained from sample learning show excellent ability to describe tumor characteristics [1], and the deep features are highly correlated with traditional features, indicating that deep features have the capabilities of mining image information [2]. Kumar D et al. [3] proposed a CAD system that uses deep features extracted from an autoencoder to classify lung nodules. Wang $\mathrm{H}$ et al. [4] presented a cascaded approach for mitosis detection that intelligently combines a CNN model and handcrafted features for breast cancer.

In this study, we developed a computer-aided differentiation method using deep feature of contrast-enhanced MR images for HCC staging. Firstly, a multiview resampling method was performed on orthogonal views (axial, coronal, sagittal) of 3D tumors, in order to generate sufficient samples for CNN training. Then, a feature extraction method based on CNN network architecture was introduced to obtain deep features that were used to characterize HCC. Finally, an ensemble classifier that fusions deep features derived from three orthogonal views was designed for HCC staging. Our method was conducted on 46 clinical HCCs which were pathologically verified.

\section{Method}

\subsection{Data and Preprocessing}

Tissue samples from our study were collected from 46 HCC patients. Gd-DTPA enhanced MRI was performed. The lesions were histologically divided into well-differentiated lesions and poorlydifferentiated lesions. The region-of-interest (ROI) was manually drawn by a physician with 10 years of clinical experience in abdomen radiology. As the 46 cases were rather small to perform deep learning, a resampling method based on three orthogonal views was adopted in order to generate a large number of training samples. The resampling technique within 3D tumor region can significantly increase the training sample [5]. For a 3D tumor, we considered the axial, coronal and sagittal views as the benchmark, and each view performed $N$ translation for each ROI, so the total number of samples for the 3D tumor was $N_{\text {all }}=N_{a} * N_{c} * N_{s}$. This procedure results in $N_{\text {all }}$ orthogonal 
observations of each ROI. As HCCs were small and image contrast was not high, only translation was used in the process of resampling.

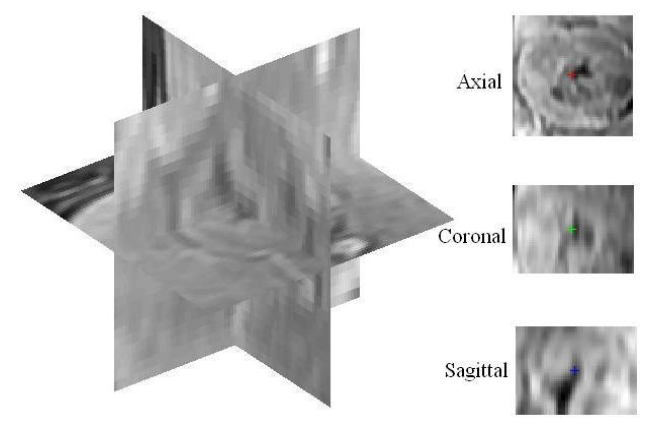

\subsection{Convolution Neural Network}

Fig. 1 Orthogonal views of a 3D tumor.

Deep learning is the most popular technology in the field of machine learning. Convolution neural network $(\mathrm{CNN})$ is one of the most important methods of deep learning [6], which convolves the original image $\mathrm{x}$ with convolution kernel $w$ to get the convolution feature maps of the image:

$$
\mathrm{y}_{n}^{i}=F\left(\sum_{j=0}^{C_{n-1}} w_{n}^{i j} * x_{n-1}^{j}+b_{n}\right)
$$

Where, $\mathrm{y}_{n}^{i}$ is the output of the $i$-th neuron of the $n$-th layer, and $x_{n-1}^{j}$ is the input of the $j$-th neuron of the (n-1)-th layer. $C_{n-1}$ is the number of $n-1$ neurons, $b$ is the bias parameter in the convolution process, and $F(\cdot)$ is the squashing function. Max-pooling is then used to reduce the connection parameters between neurons and to preserve the spatial invariance of the feature maps. After a series of convolutional and pooling, the final feature maps is obtained and connected to the fully connected layer to achieve the final classification output.

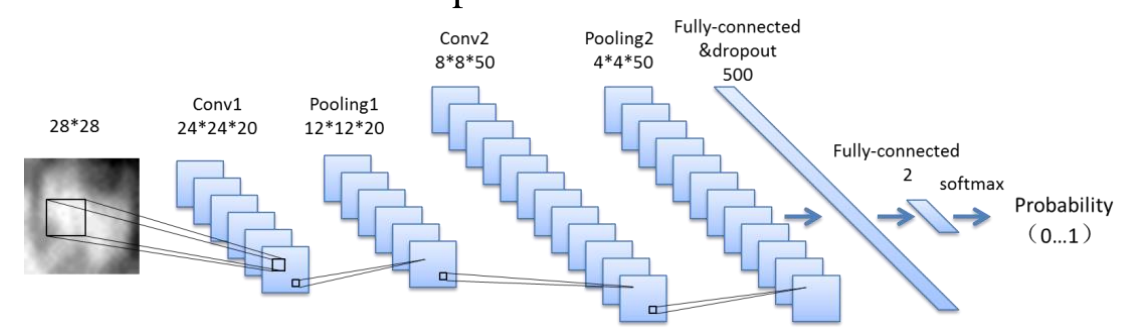

Fig. 2 Convolutional neural network architecture

In this study, our model is based on the CNN architecture, which has two convolutions, the convolution kernel is $5 \times 5$, the first convolution layer outputs 20 feature maps, the second convolution output 50 feature maps. Each convolution layer is connected with a $2 \times 2$ layer of the max-pooling. Then connected with the two-layer fully connected layer, the number of neurons was 500 and 2, respectively. Finally, the "softmax" layer output classification of the probability of identification results. An open source deep learning framework "caffe" [7] was used to implement our model, which provided the interface with the NVIDIA Graphics Processing Unit. Cuda and cuDNN achieve GPU acceleration can be used to improve training speed of the model.

\subsection{Deep Features}

Deep learning have been used to extract deep features of images. As a new feature descriptor, deep feature is a result of autonomous learning compared with the traditional morphological texture feature, which compensates for the defects in the design of manual features. In the present study, it can be found that $\mathrm{CNN}$ convolution templates have the ability to extract tumor features from low-resolution MRIs. The trained CNN network generates deep feature maps at each level of training. The feature maps of the first convolution layer in the CNN training were shown in Fig.3. Deep features derived from the last fully connected layer were chosen for the following classification. 


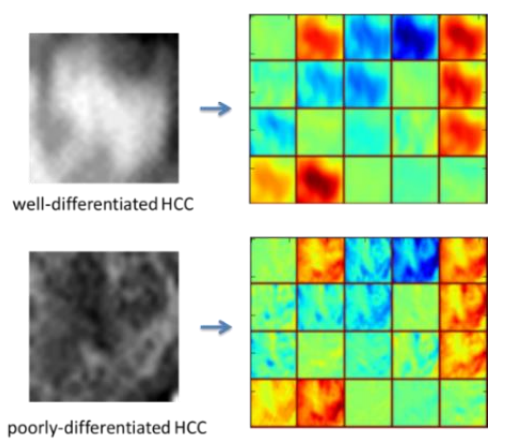

Fig. 3 Deep features derived from well-differentiated and poorly-differentiated HCCs

\subsection{Classification}

After extracting the deep features of the image, an ensemble classification was designed as shown in Fig.4. Firstly, all the three orthogonal observations of the tumor represent intrinsic tumor information. The samples of the three observations were accumulated to generate deep features for each view, respectively. Then, BP neural network (BPNN) classifier was chosen for classification. BPNN can replace the fully connected layer in CNN to classify the deep features, and then output reliable results using the Adaboost technique. Adaboost is a multi-classifier combination method, which can adaptively adjust multiple classifiers to improve classification performance.

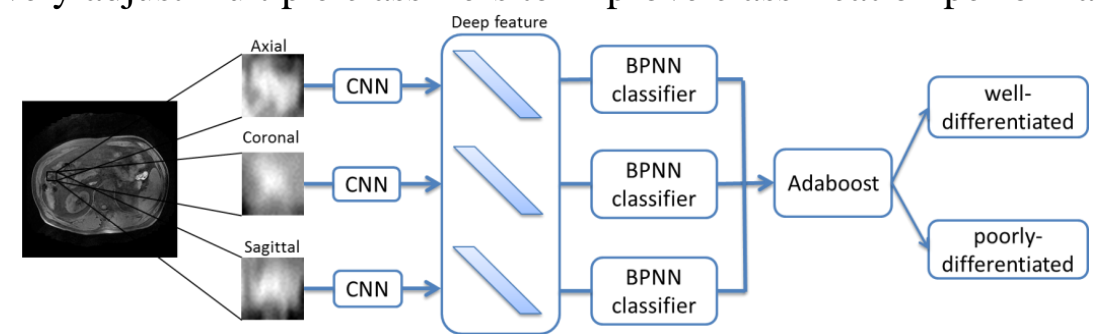

Fig. 4 flowchart based on deep feature and ensemble classification

\section{Results and Discussion}

The proposed deep learning method was compared with other machine learning methods that use texture features. Texture features including histogram features (mean, variance, skewness, kurtosis and energy), gray level co-occurrence matrix (GLCM) and gray level run-length matrix (GLRLM) were selected. LibSVM[8] and BPNN were chosen for comparison. 10-times 4-fold Cross validation was used for performance comparison, including randomly selecting 33 patients as training samples and 13 samples as test samples. The four methods use the same training samples and test samples to ensure the fair comparison. Our method uses three views to get the deep feature, and then use BPNN and adaboost ensemble classification. Test results were tabulated in Table 1. LibSVM was much better than BPNN in terms of small samples, and CNN outperformed LibSVM and BPNN. However, our approach yielded best results.

Table 1 comparison of general classification method and our method (\%)

\begin{tabular}{|c|c|c|c|}
\hline Method & Accuracy & Sensitivity & Specificity \\
\hline LibSVM & $82.50 \pm 12.70$ & $88.89 \pm 11.15$ & $74.55 \pm 22.01$ \\
\hline BPNN & $74.62 \pm 7.73$ & $73.33 \pm 22.61$ & $75.71 \pm 20.25$ \\
\hline CNN & $86.15 \pm 7.07$ & $85.00 \pm 9.46$ & $87.14 \pm 10.54$ \\
\hline Our method & $91.08 \pm 6.83$ & $89.17 \pm 7.22$ & $92.58 \pm 8.47$ \\
\hline
\end{tabular}

Compared with the results in [9], we applied the training method of deep learning to HCC. At the same time, we overcome the problem of low resolution and ROI of HCC images, and excavated the deep features of HCC, which can well characterize HCC morphology. Furthermore, more data will be acquired to evaluate the performance of the proposed method in the future work. 


\section{Conclusion}

The proposed study suggests that deep feature derived from CNN can be well described the characterization of HCC tumors, which yields best staging results than low-level features. Meanwhile, $3 \mathrm{D}$ resampling of tumor slice is an effective method to improve the sample size and capture abundant information of 3D tumors, which can be extensively used in clinical practice. Finally, the proposed ensemble classification method that combines deep features derived from three orthogonal views outperforms the state-of-the-art methods for HCC staging.

\section{References}

[1]. Nishie, A., Tajima, T., Asayama, Y., et al. Diagnostic performance of apparent diffusion coefficient for predicting histological grade of hepatocellular carcinoma. European Journal of Radiology. Vol. 80 (2011) No. 2, p. 29-33.

[2]. Xu, Y., Mo, T., Feng, Q., et al. Deep learning of feature representation with multiple instance learning for medical image analysis. IEEE International Conference on Acoustics, Speech and Signal Processing (ICASSP). Florence. Italy, 2014, p. 1626-1630.

[3]. Kumar, D., Wong, A., Clausi, D. A., et al. Lung Nodule Classification Using Deep Features in CT Images. Computer and Robot Vision. Vol. 327 (2015), p. 110-116

[4]. Wang, H., Cruz-Roa, A., Basavanhally, A., et al. Mitosis detection in breast cancer pathology images by combining handcrafted and convolutional neural network features. Medical Imaging, Vol. 1 (2014) No. 3, p. 1-8.

[5]. Ciompi, F., Hoop, B.D., Riel, S.J.V., et al. Automatic classification of pulmonary peri-fissural nodules in computed tomography using an ensemble of $2 \mathrm{~d}$ views and a convolutional neural network out-of-the-box. Medical Image Analysis. Vol. 26 (2011) No. 1, p. 195-202.

[6]. Krizhevsky, A., Sutskever, I., Hinton, G.E. et al. Imagenet classification with deep convolutional neural networks. Advances in Neural Information Processing Systems. Vol. 25 (2012) No. 2, p. 1097-1105.

[7]. Jia, Y., Shelhamer, E., Donahue, J., et al. Caffe: convolutional architecture for fast feature embedding. ACM International Conference on Multimedia. Brisbane. Australia, 2014, p. 675678.

[8]. Chang, C.C., Lin, C.J., et al. Libsvm: a library for support vector machines. ACM Transactions on Intelligent Systems \& Technology. Vol. 3 (2007) No. 27, p. 389-396.

[9]. Roth, H., Lu, L., Liu, J., et al. Improving computer-aided detection using convolutional neural networks and random view aggregation. IEEE Trans. Med. Imaging. Vol. 35 (2015) No. 5, p. $1170-1181$. 